\title{
Vom Beruf unserer Zeit für die Rechtsphilosophie
}

\author{
Gunnar Duttge*
}

\begin{abstract}
Rezension zu: David Kräft, Apriorität und Positivität des Rechts nach Kant (FUNDAMENTA JURIDICA. Beiträge zur rechtswissenschaftlichen Grundlagenforschung, Bd. 63), BadenBaden (Nomos) 2011, 130 S., brosch. 34,- €, ISBN 978-3-8329-6732-1
\end{abstract}

\section{A. „Was ist Recht?“}

Keine rechtsphilosophische Lehrbuch- oder Studienliteratur, keine auch nur ansatzweise ausgearbeitete rechtsphilosophische Theorie, in der diese Fundamentalfrage nicht wenigstens implizit im Mittelpunkt steht. Vom Begriff des Rechts ausgehend erschließen sich nahtlos alle zentralen Themen rechtsphilosophischer Aufklärung: das Abgrenzungsproblem zur Moral, der ewige Widerstreit zwischen Naturrecht und Rechtspositivismus, Wesen und Voraussetzungen der „Rechtsgeltung“, und von hier aus natürlich auch die komplexen Fragen der sog. „Rechtsanwendung“ (besser „Konkretisierung“), etwa zur Gesetzesbindung und richterlichen Rechtsfortbildung, der juristischen Argumentation, der Normentheorie sowie zur Wissenschaftlichkeit der Rechtswissenschaft(en). Wer sich freilich auf dieses Feld begibt, bewegt sich „auf vielfach gepflügtem Boden“; die bereits gewonnenen Erkenntnisse lassen sich daher nicht „ungestraft“ ignorieren (Johann Braun).

In diesem Lichte gewinnt der Umstand seine eigentliche Bedeutung, dass auch schon der große Königsberger Immanuel Kant in seiner „Einleitung in die Rechtslehre“ mit jener Ausgangsfrage angehoben hat: „Was ist Recht? “; bekanntlich versah er sie zugleich mit einer polemischen, an die Juristenzunft adressierten Zusatzbemerkung:

„Diese Frage möchte wohl den Rechtsgelehrten, wenn er nicht in Tautologie verfallen oder, statt einer allgemeinen Auflösung, auf das, was in irgendeinem Lande die Gesetze zu irgendeiner Zeit wollen, verweisen will, ebenso in Verlegenheit setzen als die berufene Aufforderung: Was ist Wabrheit? den Logiker. Was rechtens sei (quid sit iuris), d.h. was die Gesetze an einem gewissen Ort und zu einer gewissen Zeit sagen oder gesagt haben, kann er noch wohl angeben; aber, ob das, was sie wollten, auch recht sei, und das allgemeine Kriterium, woran man überhaupt Recht sowohl als Unrecht (iustum et iniustum) erkennen könne, bleibt ihm wohl verborgen, wenn er nicht eine Zeitlang jene empirischen Prinzipien verlässt, die Quellen jener Urteile in der bloßen Vernunft sucht (wiewohl ihm dazu jene Gesetze vortrefflich zum Leitfaden dienen können), um zu einer möglichen positiven Gesetzgebung die Grundlage zu errichten.

* Prof. Dr. Gunnar Duttge ist Direktor der Abteilung für strafrechtliches Medizin- und Biorecht an der Georg-August-Universität Göttingen und zugleich Stellvertretender Direktor des dortigen Zentrums für Medizinrecht. 
Eine bloß empirische Rechtslehre ist [...] ein Kopf, der schön sein mag, nur schade! dass er kein Gehirn hat. "1

Mit dieser dezidierten Abwendung von jedwedem empirisch-pragmatischen Denken im Rahmen der Rechtsbegründung, die ihren Ausgangs- und Bezugspunkt statt dessen in der „reinen Vernunft“ findet (d.h. apriorisch [= vor aller Erfahrung] verfährt), wird die Kantische Rechtsphilosophie üblicherweise als „metaphysisch“ bzw. „naturrechtlich“ klassifiziert, die einem rechtspositivistischen, der Rechtssetzung durch staatliche Instanzen kategoriale Bedeutung zuweisenden Grundverständnis zuwiderlaufe. Kant gehe es, so etwa die Deutung bei Georg Geismann, „um eine aus reiner juridisch-praktischer Vernunft stammende(n), also apriorische(n) Gesetzgebung als Quelle der Rechtsgesetze und einer darauf basierenden Politik als ausübender Rechtslehre“2. Auch Otfried Höffe hält dem „Philosophen des kategorischen Imperativs“ einen „Irrtum des Zuviels“ vor: Den Bereich des Apriori habe er zu weit gezogen, den des Empirischen dagegen zu eng. ${ }^{3}$ Manche Kritiker wie etwa Odo Marquard formulieren noch radikaler, dass sich die kantische Schule vollständig von der Wirklichkeit verabschiedet habe und „ganz und gar ins Prinzipielle: ins transzendentale Wolkentreten" übergetreten sei. ${ }^{4}$ Demgegenüber hat freilich schon Gustav Radbruch betont, dass es doch gerade Kant gewesen ist, der „den entscheidenden Schlag gegen das Naturrecht" geführt habe: Denn

„Kants Kritik der Vernunft hat gezeigt, dass die Vernunft nicht ein Arsenal [...] anwendungsreifer ethischer und ästhetischer Normen sei, vielmehr nur das Vermögen, zu solchen Erkenntnissen und Normen zu gelangen, ein Inbegriff nicht von Antworten, sondern von Fragen, [...] von Formen, die erst durch die Aufnahme eines gegebenen Stoffs, von Kategorien, die erst durch die Anwendung auf ein gegebenes Material Urteile [...] eines bestimmten Inhalts zu liefern vermögen. "5

Ganz in dieser Tradition hat es sich der Autor der hier rezensierten, unter der Betreuung des Hannoveraner Rechtsphilosophen Manfred Walther verfassten Dissertation zum Ziel gesetzt, im Wege einer „kritischen Rekonstruktion“ des kantischen Rechtsbegriffs die „vernunftnotwendige“ Positivität des Rechts zu beweisen. Die „Pointe“ der kantischen Rechtslehre liege gerade darin, dass deren apriorisches Fun-

1 I. Kant, Die Metaphysik der Sitten, Erster Teil: Metaphysische Anfangsgründe der Rechtslehre, Einleitung in die Rechtslehre, 1797/98, AB 31 f. (\$ B), in: W. Weischedel (Hrsg.), Immanuel Kant. Werke in zehn Bänden, Bd. 7, Frankfurt am Main 1983 (5. Nachdruck der Ausgabe 1956), S. 336.

2 G. Geismann, in: S. B. Byrd/J. Hruschka/J. C. Joerden (Hrsg.), Jahrbuch für Recht und Ethik Bd. 14, Berlin 2006, S. 3 (109f.).

3 O. Höffe, Kategorische Rechtsprinzipien. Ein Kontrapunkt der Moderne, 1994, S. 21.

4 O. Marquard, in: ders./H. Staudinger (Hrsg.), Anfang und Ende menschlichen Lebens, München/Paderborn 1987, S. 112.

5 G. Radbruch, Rechtsphilosophie, 3. Aufl. 1932, S. 15 f. (in: A. Kaufmann [Hrsg.], Gustav RadbruchGesamtausgabe, Bd. 2: Rechtsphilosophie II, Heidelberg 1993, S. 241). 
dament letztlich in eine positivistische Rechtsgeltungslehre münde, d.h. der Rechtspositivismus sich lückenlos a priori herleiten lasse und im Ergebnis als a priori notwendig erweise - denn:

„Ein jedes Subjekt kann a priori wissen, dass a priori berechtigte Ansprüche sich in der Wirklichkeit einer positivistischen Relativierung unterwerfen müssen, um dauerhaft verwirklicht werden zu können; wenn es mit Kant darauf ankommt, dass die Subjekte in den rechtlichen Zustand, den sie a priori begründen sollen, auch tatsächlich eintreten, damit jeder seines Rechts teilhaftig werde, dann ist Effektivität nach Kant Begriffsmerkmal von Recht"6.

\section{B. Beweisführung für die „Positivismus-These“}

Diese am vorherrschenden Verständnis der Kantischen Rechtslehre gemessen wahrlich überraschende Volte untermauert Kräft im Wesentlichen mit zwei Argumenten: Zum einen habe Kant ein Widerstandsrecht gegen die Staatsgewalt (i.S. einer gewaltsamen Auflehnung gegen das herrschende Regime) kategorisch ausgeschlossen; dieses „Revolutionsverbot“ werde von ihm als „praktisches Vernunftprinzip“ eingeführt und habe damit den Geltungsanspruch eines „kategorischen Imperativs“. Wenn aber der bloßen Existenz eines rechtlichen Zustands unabhängig von seiner Ausgestaltung im Detail stets absoluter Geltungsvorrang zukomme, dann werde die Bedeutung des a priori begründeten Rechtsbegriffs „als Kriterium für die Verbindlichkeit des positiven Rechts vergleichgültigt, seine kategoriale Bedeutung in der faktischen Errichtung des rechtlichen Zustandes aufgehoben “7. Oder etwas schlichter formuliert: Wenn das „Vernunftnaturrecht“ dem Einzelnen selbst in der Situation eines tyrannischen Regimes kein Recht zur tätigen Selbsthilfe verleiht, dann bleibt es hinsichtlich seines praktischen Geltungsanspruchs stets virtuell und das jeweils gesetzte Recht ohne Rücksicht auf seine materielle „Qualität“ (d.h. selbst bei „gesetzlichem Unrecht“) davon unberührt. Die Parallele zu Radbruchs Rechtspositivismus vor seiner „naturrechtlichen Wende“ (bzw. vor „Neuakzentuierung“ seines „Wertrelativismus“ $)^{8}$ drängt sich geradezu auf, eindringlich in seiner „Rechtsphilosophie“ aus dem Jahre 1932 zum Ausdruck gebracht:

„Wir verachten den Pfarrer, der gegen seine Überzengung predigt, aber wir verehren den Richter, der sich durch sein widerstrebendes Rechtsgefühl in seiner Gesetzestreue nicht beirren lässt; denn [...] das Gesetz hat nicht nur als

6 D. Kräft, Apriorität und Positivität des Rechts nach Kant, 2011, S. 18 f. und 116.

7 Kräft (Fn 6), S. 62 f.

$8 \mathrm{Zu}$ diesem bekanntlich sehr lebhaften Streit innerhalb der Radbruch-Forschung siehe einerseits F. von Hippel, Gustav Radbruch als rechtsphilosophischer Denker, Heidelberg 1951, insbes. S. 36, 99 („Damaskuserlebnis“), andererseits W. Hassemer, in: Arthur Kaufmann (Hrsg.), Gustav Radbruch-Gesamtausgabe, Bd. 3: Rechtsphilosophie III, Heidelberg 1990, S. 1, 6 ff.; Arthur Kaufmann, Über Gerechtigkeit, Köln et al. 1993, S. 473 f. 
Niederschlag der Gerechtigkeit seinen Wert, sondern auch als Bürgschaft der Rechtssicherheit. [...] Auch wenn er, weil das Gesetz es so will, aufhört, Diener der Gerechtigkeit zu sein, bleibt er noch immer Diener der Rechtssicherheit"9.

Das zweite Argument zieht eine Verbindungslinie von der „Rechtslehre“ zu Kants politischer Philosophie, die nach Auffassung von Kräft in solcher Weise „methodisch zusammenhängen “, dass letztere die erstgenannte „inhaltlich ergänzt ${ }^{10}$. Konkret ist damit das berühmte „Volk von Teufeln“-Argument aus Kants Friedensschrift in den Blick genommen, wonach auch rationale Egoisten (moralische „Teufel“) bereits aus „instrumenteller Vernunft“ („,wenn sie nur Verstand haben“) hinreichend motiviert seien, das Bestehen einer positiven Rechtsordnung gutzuheißen (das „Problem der Staatserrichtung " zu lösen). ${ }^{11}$ Da Kant in diesem Kontext auf jedwede transzendentale Herleitung verzichte und anstelle dessen ausschließlich auf einen „Natur“-Verlauf rekurriere, durch welchen - bei entsprechend „guter Organisation des Staates“ (scil.: innerhalb einer „republikanischen Verfassung “) ${ }^{12}$ - die „selbstsüchtigen Neigungen“ und die aus ihnen hervorgehenden „Kräfte“ sich in ihrer „zerstörenden Wirkung “ gegenseitig aufhielten, zeige sich hier ein „im Kern empirisch-konsequentialistischer Gedankengang“13. Dass auf diese Weise die „Achtung vor der Gesetzgebung der praktischen Vernunft als Motivationsquell der Subjekte zur Errichtung und Bewahrung eines bürgerlichen und also Friedenszustands für überflüssig erklärt“ werde,${ }^{14}$ stehe jedoch nicht im Widerspruch zur (primär) apriorischen Rechtsbegründung, sondern füge sich vielmehr in den „Gesamtzusammenhang des kantischen Theoriegebäudes“ ein. Denn es werde hierdurch ein Problemlösungsansatz „konsequent weitergeführt“, der sich schon in der „Rechtslehre“ finde: So leite der dortige $\$ 42$ das „Postulat des öffentlichen Rechts“ (scil.: „Du sollst in einen rechtlichen Zustand übergehen ") einzig und allein aus der Faktizität der Lebensbedingungen her, und zwar aus dem „unvermeidlichen Nebeneinander“ der Subjekte auf begrenztem Lebensraum sowie aus der „Neigung“ des Menschen zur Gewaltsamkeit, von der wir nur aus (Selbst-)Erfahrung wissen können. Wenn Kant hieraus folgere, dass ein

9 Radbruch (Fn 5), S. 83 f. (Gesamtausgabe, S. 316).

10 Kräft (Fn 6), S. 117.

11 I. Kant, Zum ewigen Frieden, 1795, in: W. Weischedel (Hrsg.), Werke in zehn Bänden, Bd. IX, Frankfurt am Main 1983, S. 191, insbes. $223 \mathrm{f}$.

12 Von Kant in der Friedensschrift wie folgt definiert: „Die erstlich nach Prinzipien der Freiheit der Glieder einer Gesellschaft (als Menschen); zweitens nach Grundsätzen der Abhängigkeit aller von einer einzigen gemeinsamen Gesetzgebung (als Untertanen); und drittens, die nach dem Gesetz der Gleichheit derselben (als Staatsbürger) gestiftete Verfassung - die einzige, welche aus der Idee des ursprünglichen Vertrags hervorgeht, auf der alle rechtliche Gesetzgebung eines Volkes gegründet sein muss“" (Zum ewigen Frieden [o. Fn 11], S. 204); vertiefend W. Kersting, in: O. Höffe (Hrsg.), Immanuel Kant. Zum ewigen Frieden, Berlin 1995, S. 87 ff.

13 Kräft (Fn 6), S. 104.

14 Kräft (Fn 6), S. 100 f. unter Verweis auf Kant, Zum Ewigen Frieden (Fn 11), S. 224: „Denn es ist nicht die moralische Besserung der Menschen [erforderlich], um [...] den Friedenszustand, in welchem Gesetze Kraft haben, herbei[zu]führen“. 
Subjekt deshalb nicht bis zur „wirklichen Feindseligkeit“ zuwarten darf (!), so werde auf diese Weise eine stringente, sehr an Hobbes erinnernde ${ }^{15}$ Beweisführung a posteriori präsentiert, die jenem Kant gerne zugeschriebenen „normativen Rigorismus“ zuwiderlaufe. ${ }^{16}$

Man wird Kräft voller Bewunderung attestieren müssen, dass er hiermit eine prima vista ungewohnte Hypothese mittels eines innovativen Argumentationsganges auf der Basis akribischer Textexegese beachtenswert plausibilisiert hat. Dass die geknüpften Zusammenhänge und insbesondere die daraus gezogenen Schlussfolgerungen aber zwingend sind, dürfte sich kaum annehmen lassen. Das gilt insbesondere für das zweite, die postulierte „Neutralität der kategorischen Normativität“17 auf die Friedensschrift stützende Argument. Denn schon denklogisch löst sich dadurch, dass Kant für die Rechtsbegründung noch einen weiteren Weg mit „sparsameren“ (nicht-transzendentalen) Anforderungen skizziert hat, der vorgängig explizierte apriorische Rechtsbegriff („Inbegriff der Bedingungen, unter denen die Willkür des einen mit der Willkür des anderen nach einem allgemeinen Gesetze der Freiheit zusammen vereinigt werden kann“) ${ }^{18}$ nicht in Luft auf. Vielmehr bleibt das ,allgemeine Rechtsprinzip“ 19 wie das daraus hervorgehende „allgemeine Rechtsgesetz“20 von allem Wirken der hoheitlichen Rechtssetzungsorgane unberührt und fließt kraft seiner „unbedingten Notwendigkeit“ 21 gerade umgekehrt - wenngleich in unbestimmtem Ausmaß - in den unhintergehbar vom Prinzip der „Freiheitssicherung “22 geprägten Rechtsbegriff ein, wie schon die Friedensschrift selbst in ihrem Anhang unmissverständlich verdeutlicht: Denn „[...] wenn es keine Freiheit und [kein] darauf gegründetes moralisches Gesetz gibt, sondern alles, was geschieht oder geschehen kann, bloßer Mechanism[us] der Natur ist, so ist [...] der Rechtsbegriff ein sachleerer Gedanke“23. Und im unmittelbaren Anschluss wird eine „unumgängliche“ Verbindung jenes Rechtsbegriffs mit der Politik in den Blick genommen, mehr noch ist sogar davon die Rede, ihn „zur einschränkenden Bedingung der letzteren zu erheben“24,

15 Vgl. W. Kersting, Wohlgeordnete Freiheit. Immanuel Kants Rechts- und Staatsphilosophie, Paderborn 1993, S. 330: „hobbesianisierende Skizze“.

16 Kräft (Fn 6), S. 19 und $44 \mathrm{ff}$.

17 Kräft (Fn 6), S. 19.

18 Kant, Die Metaphysik der Sitten (Fn 1), $\mathbb{~ B ~ ( S . ~ 3 3 7 ) . ~}$

19 Kant, Die Metaphysik der Sitten (Fn 1), $\mathbb{C}$ (S. 337): „Eine jede Handlung ist recht, die oder nach deren Maxime die Freiheit der Willkür eines jeden mit jedermanns Freiheit nach einem allgemeinen Gesetze zusammen bestehen kann“.

20 Kant, Die Metaphysik der Sitten (Fn 1), $\mathbb{S C}$ (S. 338): „Handle äußerlich so, dass der freie Gebrauch Deiner Willkür mit der Freiheit von jedermann nach einem allgemeinen Gesetze zusammen bestehen könne“.

21 Kant, Zum ewigen Frieden (Fn 11), S. 239.

22 Geismann (Fn 2), S. 109: „Das Recht ist [...] nichts anderes als Freiheitssicherungsordnung“.

23 Kant, Zum ewigen Frieden (Fn 11), S. 232 [Hervorhebung G.D.].

24 Ebd. 
was sich zusammen mit dem nachfolgenden Ideal eines „moralischen Politikers“25 (ohne damit jedoch eine „Königsherrschaft der Philosophen“ zu verbinden) ${ }^{26}$ gar nicht anders als eine programmatische Positionierung im kantischen Sinne auffassen lässt.

Wie Kräft keineswegs bestreitet, bleibt das „vernünftige Privatrecht“ zweifelsohne materieller Fixpunkt für das positive Recht, das sich im Falle seiner Defizienz „durch Reform schrittweise anzugleichen " habe. ${ }^{27}$ Schon der eingangs zitierten Sentenz über die „Hirnlosigkeit“ einer „bloß empirischen Rechtslehre“ ist die klare Prioritätsregel inhärent: „Die positive Gesetzgebung hat sich nach der natürlichen zu richten, nicht umgekehrt"!28 Streitig kann somit nur das Wie, nicht aber das Ob einer solchen Priorität sein. Dass diese - rechtsmethodologisch gesprochen - ausschließlich de lege ferenda und in keinster Weise schon de lege lata ihre Wirkung entfaltet, ergibt sich auch aus dem „Revolutionsverbot“ durchaus nicht zwingend. Denn dieses hat seinen Grund ersichtlich darin, dass der „aktiv-gewaltsame Widerstand“ die Staatsgewalt als solche negiert und damit den „status civilis“ in Gänze beseitigt, ${ }^{29}$ indem sich das Volk extralegale Exekutivbefugnisse anmaßt. Die „negative Weigerung“ zur Befolgung der Gesetze - der „zivile Ungehorsam “ in moderner Terminologie - ist hingegen keineswegs verboten: Vielmehr wäre die willfährige Befolgung unmoralischer Gesetze nach Kant sogar „ein sicheres Zeichen [dafür], dass das Volk verderbt, seine Repräsentanten [er]käuflich und das Oberhaupt in der Regierung durch seinen Minister despotisch, dieser selbst aber ein Verräter des Volkes sei“"30. Diese enge Begrenzung des „Widerstandsrechts“ sieht Kräft sehr wohl und konstatiert im Ansatz zutreffend, dass bei friedlichem Gesetzesungehorsam „dem staatlichen Regime [...] die Effektivität seiner Gesetzgebung in einem konkreten Fall versagt“ bleibe. ${ }^{31}$ Was hier allerdings nicht präzise genug zum Vorschein tritt: Es handelt sich dabei mitnichten um eine „Effektivitätsgrenze“ infolge mangelnder faktischer Durchsetzungsmacht, sondern um eine solche aus übergeordnetem Rechtsgrund. Gebietet ein positives Gesetz etwas, „was an sich böse (dem Sittengesetz unmittelbar zuwider) ist“,

25 Kant, Zum ewigen Frieden (Fn 11), S. 233: „Der moralische Politiker wird es sich zum Grundsatz machen: wenn einmal Gebrechen in der Staatsverfassung [...] angetroffen werden, [...] so sei es Pflicht, vornehmlich für Staatsoberhäupter, dahin bedacht zu sein, wie sie, sobald wie möglich, gebessert und dem Naturrecht, so wie es in der Idee der Vernunft uns zum Muster vor Augen steht (!), angemessen gemacht werden könne" [Hervorhebung G.D.].

26 Dazu eingehend V. Gerhardt, in: Höffe (Fn 12), S. $171 \mathrm{ff}$.

27 Kräft (Fn 6), S. 116; auf S. 59 begegnet die zutreffende Frage, wie „Vollzugslücken des vernünftigen Privatrechts sich rechtfertigen lassen".

28 O. Höffe, in: ders. (Hrsg.), Immanuel Kant. Metaphysische Anfangsgründe der Rechtslehre, Berlin 1999 , S. 41, 43.

29 Auf die Unmöglichkeit einer ,juridischen Befugnis zur Rebellion gegen den status civilis“ abhebend: R. Keil, in: J. Bung/B. Valerius/S. Ziemann (Hrsg.), Normativität und Rechtskritik, ARSP-Beiheft Nr. 114, 2007, S. 45, 64 m.w.N.

30 Kant, Die Metaphysik der Sitten (Fn 1), Allgemeine Anmerkung A (S. 441 f.).

31 Kräft (Fn 6), S. 70 ff., 74. 
dann „darf und soll“ (!) ihm „nicht gehorcht werden“32. Wenn auf diese Weise aber einzelne Äußerungen der Staatsgewalt normativ suspendiert werden können, lässt sich von einem „Rechtspositivismus“ jedenfalls in Reinform nicht mehr sprechen. In diesem Sinne hat somit Höffe mit Recht betont, dass Kant fern einer Positionierung innerhalb simpler Schwarz-Weiß-Schemata „weder der positiven noch der überpositiven Rechtsbetrachtung ein Exklusivitätsrecht ein[räumt]“33. Aus der Perspektive der Jurisprudenz wird diese Konvergenz der beiden „Reiche“ an der auffällig kantischen Prägung des Grundgesetzes ${ }^{34}$ und insbesondere der Menschenwürdegarantie des Art. 1 GG greifbar. Die Frage ist allein, wo genau und anhand welchen Kriteriums die Trennlinie verläuft, sofern das normativ vorgeordnete „Vernunftnaturrecht“ überhaupt noch einen Inhalt hat und nicht bloß reine Form (mit „wechselnden Inhalten“) ${ }^{35}$ ist. Zu dieser Frage wird man allerdings in der kantischen „Rechtslehre“ keine (eindeutige) Antwort finden.

\section{Aufgabe einer Rechts-Wissenschaft}

Ohne einen „archimedischen Punkt“, ohne irgendeine „metaphysische Verankerung “ gerät jeder Versuch einer „Navigation“ innerhalb des Rechts zu einer ziellosen Irrfahrt. ${ }^{36}$ Dies ist der Grundgedanke einer „Rechtsmetaphysik“ gleichsam als richtungweisender „Kompass“ aufgeklärter Jurisprudenz, was mitnichten eine simplifizierende Ableitung scheinbar feststehender konkreter Naturrechtsgehalte (noch dazu mit dem Anspruch auf „Letztbegründung“) bedeutet. ${ }^{37}$ Will das Recht nicht nur aus Macht resultierende Zwangsordnung, sondern Ausdruck eines wertbezogen begründeten Sollens sein, was seinen eigentümlichen Anspruch von „Objektivität“ erst verständlich macht, so muss eine jede Rechtstheorie die Abhängigkeit des positiven Rechts von einer vorgängigen Rechtsmoral zugestehen. ${ }^{38}$ Mit ihr steht und fällt also die fundamentale Legitimation von „Recht“, das diesen Namen noch verdient und nicht bloße Verbrämung willkürlicher Regeln der Macht ist. Was durch die anhaltende „Entmoralisierung“ des Rechts letztlich verlorengeht, findet sich bei Naucke/ Harzer treffend benannt:

32 I. Kant, Die Religion innerhalb der Grenzen der bloßen Vernunft, in: W. Weischedel (Hrsg.), Immanuel Kant. Werke in zehn Bänden, Bd. 7, Frankfurt am Main 1983 (5. Nachdruck der Ausgabe 1956), S. 758, Fn. *.

33 Höffe (Fn 28), S. 43.

34 Dazu etwa H. Bielefeldt, in: W. Brugger (Hrsg.), Legitimation des Grundgesetzes aus Sicht von Rechtsphilosophie und Gesellschaftstheorie, Baden-Baden 1996, S. $47 \mathrm{ff}$.

35 Zur Idee eines „Naturrechts mit wechselndem Inhalt“ s. schon Radbruch, Rechtsphilosophie (Fn 5), S. 241; ihm folgend Arthur Kaufmann, Rechtsphilosophie, München 1997, S. 26; ausführlich dazu S. Grote, Auf der Suche nach einem „dritten Weg“, Baden-Baden, 2008.

36 Dazu näher G. Duttge/M. Löwe, in: Byrd/Hruschka/Joerden (Hrsg.), Jahrbuch (Fn. 2), S. 351 ff., insbes. S. $382 \mathrm{f}$.

37 Zur Renaissance des „Perfektionismus“ vgl. etwa H. Pauer-Studer, in: R. Schmücker/U. Steinvorth (Hrsg.), Gerechtigkeit und Politik. Philosophische Perspektiven, Berlin 2002, S. 77 ff.

Zutr. Höffe (Fn 3), S. 74. 
„Die Schwächung aller Formen von Metaphysik schwächt auch die Metaphysik der Freiheit, lässt von ihr nur die äußere Willkür im Handlungsraum, das opportune, utilitäre Verhalten übrig. [...] Was die Suche nach absolut richtigem Recht gerade angereizt hatte, [war] die rechtliche Sicherung der Mindestbedingungen für ein menschenwürdiges Leben. Streicht man die Metaphysik des Rechts [...], so [...] entsteht eine uneingestandene Ordnungslehre als Reaktion auf die Entwicklung, die die Willkür durch das Recht verstärkt und die nun eine Willkür-Ordnung auf die nächste Willkür-Ordnung folgen lässt. [...] Diese Ordnungslehre ist schwach, ihr Begriff von (zweckmäßiger) Ordnung formal; es kann nicht angegeben werden, welchen Inhalt diese Ordnung haben muss. [...] Dass Rechtslehre dann nur noch polizeiliche Regellehre ist, gesteht sie sich nicht ein" 39 .

Rechts-Wissenschaft im wahren Sinne erschöpft sich nicht in der bloß rezeptiven Kenntnis (einzelner Teile) des positiven Rechts („Rechtskunde“); sie hat vielmehr das „systematische Verstehen des Rechtsstoffes aus Prinzipien“40 zum Gegenstand bis hin zum Ergründen der „unwandelbaren Prinzipien [...] zu aller positiven Gesetzgebung " 41 auf epistemisch höchster Stufe. Es sollte der heutigen akademischen Rechtslehre im Zeitalter „post Bologna“ sehr zu denken geben, dass Kant die vorstehende Aufgabe bei den „schriftgelehrten Juristen“ nicht in guten Händen gesehen hat: weil diese „die Gesetze der Sicherung des Mein und Dein (wenn sie [...] als Beamte der Regierung verfahren) nicht in der Vernunft, sondern im [...] höchsten Orts sanktionierten Gesetzbuch "suchen. ${ }^{42}$ Der Jurist,

„der die Waage des Rechts und [da]neben [...] auch das Schwert der Gerechtigkeit sich zum Symbol gemacht hat, bedient sich gemeiniglich des letzteren, nicht um etwa bloß alle fremde Einflüsse von dem ersteren abzuhalten, sondern, wenn die eine Schale nicht sinken will, [um] das Schwert mit hinein zu legen (vae victis), ${ }^{43}$ wozu der Jurist, der nicht zugleich [...] Philosoph ist, die größte Versuchung hat". ${ }^{44}$

$\mathrm{Zu}$ seinen Pflichtengehör es aber sehr wohl, nicht nur anzugeben, „was die Gesetze an einem gewissen Orte und zu einer gewissen Zeit sagen oder gesagt haben“, sondern auch das „allgemeine Kriterium, woran man überhaupt Recht sowohl als Un-

39 W. Naucke/R. Harzer, Rechtsphilosophische Grundbegriffe, 5. Aufl. 2005, Rn 267.

40 Höffe (Fn 28), S. 45.

41 Kant, Die Metaphysik der Sitten (Fn 1), $\mathbb{~ A ~ ( S . ~ 3 3 6 ) . ~}$

42 I. Kant, Der Streit der Fakultäten, 1798, in: W. Weischedel (Hrsg.), Werke in zehn Bänden, Bd. IX, Frankfurt am Main 1983, S. 261, 287.

43 Vae victis (,,Wehe den Besiegten!") ist ein lateinischer Ausspruch des gallischen Heerführers Brennus, der vermutlich auf einen Bericht des Römers Titus Livius zurückgeht.

44 Kant, Zum ewigen Frieden (Fn 11), S. 228. 
recht [...] erkennen könne“45. Dazu bedarf es aber „freier Rechtslehrer“, die das Volk in Ansehung des Staates, dem es angehört, über seine Pflichten und Rechte öffentlich belehren; ${ }^{46}$ der wahre Rechtslehrer nimmt nicht Partei für partikulare Interessen, sondern „für die Vernunft“47. Eine reflektierte „Volksaufklärung“ via moderner Massenmedien wird man heute als aussichtsloses Unterfangen ansehen müssen; vielversprechender dürfte vielversprechender das Zurverfügungstellen von Literatur sein, die allen Interessierten - insbesondere auch den jungen Jurastudierenden - die Chance eröffnet, sich frei von kurzsichtigen praktisch-utilitaristischen Zwecken in Denkvermögen (Logik), Sprache und Werthaltung zu schulen. Das vorliegende Werk, eine „Dissertation“ im eigentlichen und besten Sinne, ist hierfür in höchstem Maße geeignet!

45 Oben Fn 1.

46 Kant, Der Streit der Fakultäten (Fn 42), S. 362 f.

47 V. Gerhardt, in. Höffe (Fn 12), S. 176 f. 